\title{
Implications, consequences and interpretations of generalized entropy in the cosmological setups
}

\author{
H. Moradpour* \\ Research Institute for Astronomy and Astrophysics of Maragha (RIAAM), \\ P.O. Box 55134-441, Maragha, Iran
}

\begin{abstract}
Recently, it was argued (Eur. Phys. J. C 73, 2487 (2013)) that the total entropy of a gravitational system should be related to the volume of system instead of the system surface. Here, we show that this new proposal cannot satisfy the unified first law of thermodynamics and the Friedmans equation simultaneously, unless the effects of dark energy candidate on the horizon entropy are considered. In fact, our study shows that some types of dark energy candidate may admit this proposal. Some general properties of required dark energy are also addressed. Moreover, our investigation shows that this new proposal for entropy, while combined with the second law of thermodynamics (as the backbone of Verlinde's proposal), helps us in providing a thermodynamic interpretation for the difference between the surface and bulk degrees of freedom which, according to Padmanabhan's proposal, leads to the emergence of spacetime and thus the universe expansion. In fact, our investigation shows that the entropy changes of system may be equal to the difference between the surface and bulk degrees of freedom falling from surface into the system volume. Briefly, our results signal us that this new proposal for entropy may be in agreement with the thermodynamics laws, the Friedmann equation, Padmanabhan's holographic proposal for the emergence of spacetime and therefore the universe expansion. In fact, this new definition of entropy may be used to make a bridge between Verlinde's and Padmanabhan's proposals.
\end{abstract}

\footnotetext{
*h.moradpour@riaam.ac.ir
} 


\section{INTRODUCTION}

The Bekenstein entropy is the backbone of study the thermodynamic aspects of Einstein field equations [1, 2]. Indeed, thanks to Jacobson unique work [3], we can take into account the Einstein field equations as a thermodynamical equation of state. His approach also provides a suitable framework for finding out the thermodynamic equation of state corresponding to the gravitational field equations in other theories of gravity [4]. Since the observed universe is dynamics [5], described by Friedmann-Lemaitre-Robertson-Walker (FLRW) metric, the investigation of mutual relation between thermodynamics laws and Friedman equation, governing the universe evolution, is important. In fact, such generalizations have been addressed by many authors in vast cosmological setups [ $[6-14]$.

There are two definitions for the temperature of apparent horizon of FLRW universe [6 9, 15, 16], called the Hayward-Kodama temperature [6 8] and the Cai-Kim temperature [9, 15], and both of them, whenever they are combined with the Bekenstein entropy relation, are in agreement with the Friedmann equation as well as the unified first law of thermodynamics. It is useful to note here that although the Bekenstein entropy is in line with the current accelerating phase of universe expansion and the second and generalized second laws of thermodynamics [17, 18], some authors show that a dark energy candidate, due to its unknown nature, may modify the horizon entropy (the Bekenstein limit in the Einstein general relativity framework) [19 23]. In their setups, the Friedmann equation and the second law of thermodynamics are still valid simultaneously [19 23]. Finally, it is useful to mention here that one may get the universe evolution equation in various theories of gravity (the corresponding Friedmann equation), by taking into account the horizon entropy relation together with the Hayward-Kodama temperature and applying the unified first law of thermodynamics on the apparent horizon of FLRW universe [24].

Verlinde shows that the second law of thermodynamics (tendency of systems to increase

their entropy [25]) may lead to the emergence of spacetime and gravity [26]. The entropyarea relation (the Bekenstein entropy for General relativity) plays a key role in this theory which attracts more attempts to itself [27-48]. In another approach, Padmanabhan argues that the difference between the surface and bulk degrees of freedom is proportional to the volume changes of system leading to the emergence of spacetime and thus the universe expansion [49]. Indeed, the positive difference between the surface and bulk degrees of 
freedom yields the spacetime emergence and the Friedmann equations in various theories of gravity [49 53]. It is also useful to note here that the Padmanabhan argument claims that the Cai-Kim temperature is more suitable option for the temperature of matter fields enclosed by the apparent horizon of FLRW universe [49]. Now, one can ask that why is the difference between the surface and bulk degrees of freedom positive, leading to the emergence of spacetime and universe expansion? Moreover, bearing the Verlinde argument in mind, may one relate the positive difference between the surface and bulk degrees of freedom to the second law of thermodynamics? Briefly, what is the relation between the thermodynamic laws and Padmanabhan's proposal?

Recently, Tsallis and Cirto proposed a new expression for the horizon entropy of Schwarzschild black hole, and therefore the entropy-area relation [54]. Indeed, they have considered the general formalism of non-additive entropy, applied that to the Schwarzschild horizon and obtained that the horizon entropy is related to the volume confined by it, instead of its surface area [54]. Moreover, It was shown that if one uses the Weyl-WignerGroenewold-Moyal formalism of deformation quantization, then the apparent horizon entropy of radiation and dust dominated quantum universes is proportional to the universe volume instead of its surface area [55]. Therefore, both the non-extensive statistics and quantum cosmology theories suggest that the horizon entropy is related to the system volume instead of its surface area. Therefore, since it was shown that the Einstein field equations are in line with thermodynamics if the horizon entropy be proportional with its surface $[2-4,6-9,15,16]$, it seems that this new hypothesis for the entropy-area relation, given by both quantum cosmology considerations [55] and non-extensive statistics [54], leads to an inconsistency in the mutual relation between the Einstein field equations and the thermodynamics laws. This inconsistency necessitates us to more investigate the relation between the non-extensive thermodynamics and the gravitational field equations. In refs. [56 58], authors show the Tsallis et al. entropy, called the generalized entropy, may be related to the bulk viscosity of cosmological fluids leading to modify the Friedmann equation. It is also pointed out that the entropy of holographic screen with entangled bits meets the generalized entropy relation and may confirm Verlinde's hypothesis of gravity [59]. Moreover, it seems that the generalized entropy, whenever it is combined with Verlind's formalism, modifies gravitational coupling constant and thus the acceleration formula [60, 61]. More studies on the relation between the generalized entropy and various aspects of cosmos can be found in 
62 64].

Therefore, based on some previous works, the Bekenstein entropy (as the geometrical entropy of horizon) is equal to the maximum entropy of fields confined by the system boundary [1, 2]. Now, since there are some works showing that a dark energy candidate may modify the horizon entropy [19 23], can one make a relation between the energy density of dark energy candidate and the general entropy relation? Here, we are going to investigate the possibility of establishing a relation between these attempts and the generalized entropy proposed by Tsallis et al. [54], which is also supported by the quantum cosmology considerations [55]. The latter may lead us to find out a profile density for the dark energy candidate. Moreover, we are eager to study the relation between the second law of thermodynamics (tendency of systems to increase their entropy) as the backbone of Verlinde's proposal, the generalized entropy and Padmanabhan's hypothesis about the origin of spacetime and the universe expansion. In other words, we try to show that the generalized entropy, combined by the second law of thermodynamics, is in line with Padmanabhan's proposal. In addition, we show that the combination of the second law of thermodynamics and the generalized entropy may lead to an acceptable thermodynamic interpretation for the positive difference between the surface and bulk degrees of freedom.

In order to present our work, we organize the paper as follows. Bearing the Cai-Kim temperature in mind, we apply the unified first law of thermodynamics to the apparent horizon of FLRW metric, and get a relation between the horizon entropy and the energy density of fluid enclosed by horizon, in the next section. We also point out the results of considering the generalized and Bekenstein entropies in the obtained relation. In addition, by making a connection between the generalized entropy, the Bekenstein entropy and the energy density of the dark energy candidate, we get a relation for the energy density of dark energy candidate. Finally, we also study some general behavior of the obtained dark energy candidate. In section (III), we investigate the relation between the generalized entropy, the second law of thermodynamics (as the backbone of Verlinde's proposal) and Padmanabhan's proposal. We show that the generalized entropy may be used to build a bridge between the second law of thermodynamics and the positive difference between the surface and bulk degrees of freedom (as the key point of Padmanabhan's proposal). The last section is devoted to a summary and concluding remarks. Throughout this paper we also set $G=\hbar=$ $c=k_{B}=1$ for the sake of simplicity. 


\section{THERMODYNAMICS, FRIEDMANN EQUATION, THE GENERALIZED ENTROPY AND DARK ENERGY}

Consider a FLRW metric

$$
d s^{2}=-d t^{2}+a^{2}(t)\left[\frac{d r^{2}}{1-\kappa r^{2}}+r^{2} d \Omega^{2}\right],
$$

in which $a(t)$ is scale factor, and $\kappa=-1,0,1$, called the curvature constant, points to the open, flat and closed universes, respectively [5]. The radii of marginally trapped surface, called the apparent horizon, is defined as

$$
\partial_{\alpha} \zeta \partial^{\alpha} \zeta=0 \rightarrow r_{A}
$$

where $\zeta=a(t) r$, and by some calculations one gets

$$
\tilde{r}_{A}=a(t) r_{A}=\frac{1}{\sqrt{H^{2}+\frac{\kappa}{a(t)^{2}}}}
$$

for the physical radii of apparent horizon $\left(\tilde{r}_{A}\right)[6]$, 12, 13]. If this spacetime be filled by a prefect fluid with energy-momentum tensor $T^{\nu}{ }_{\mu}=\operatorname{diag}(-\rho, p, p, p)$, the corresponding continuity equation can be written as

$$
\dot{\rho}+3 H(\rho+p)=0,
$$

where $\rho$ and $p$ are the energy density and pressure of the assumed source. $H \equiv \frac{\dot{a}}{a}$ is also called the Hubble parameter. Since it is argued that the apparent horizon can be considered as a proper causal boundary for the FLRW spacetime [6 8, 12, 13], we take into account it as the causal boundary. The energy amount crossing the apparent horizon during the universe expansion $\left(\delta Q^{m}\right)$ is defined as

$$
\delta Q^{m}=A\left(T_{a}^{b} \partial_{b} \zeta+W \partial_{a} \zeta\right) d x^{a}
$$

where $W=\frac{\rho-p}{2}$ and $\zeta=\tilde{r}_{A}$ are the work density and apparent horizon radii, respectively [10]. After some calculations and using Eq. (44), one obtains [22, 23]

$$
\delta Q^{m}=-3 V H(\rho+p) d t=V d \rho .
$$

In deriving this equation, we adopt $V=\frac{4 \pi}{3} \tilde{r}_{A}^{3}$ together with the Cai-Kim approach, in which $d \zeta \approx 0$, in the infinitesimal time $d t,[9]$. Bearing the Clausius relation in mind $\left(T d S_{A}=\delta Q^{m}\right)$ 
[25], we get

$$
d \rho=-\frac{T}{V} d S_{A}
$$

in that $S_{A}$ is the horizon entropy, and mines sign comes from the universe expansion [9, 10, 21 23]. Now, for a theory in which $S_{A}=S_{A}(A)$, since the Cai-Kim temperature meets the $T=\frac{1}{2 \pi \tilde{r}_{A}}$ relation [9, 10, 15, 16], one reaches

$$
\rho=-6 \int \frac{S_{A}^{\prime}(A)}{A^{2}} d A
$$

where ' denotes derivative with respect to $A$. It is useful to note here that this result is also obtained if one uses the Hayward-Kodama temperature [24]. If we insert Bekenstein relation $\left(S_{B}=\frac{A}{4}\right)$, as the apparent horizon entropy, into this equation and take integral from the RHS, we get

$$
\frac{1}{\tilde{r}_{A}^{2}}=\frac{8 \pi}{3} \rho
$$

which can finally be rewritten as

$$
H^{2}+\frac{\kappa}{a^{2}}=\frac{8 \pi}{3} \rho
$$

by helping Eq. (3). Indeed, it is nothing but the Friedmann equation [5, 9 11]. Briefly, one can use the unified first law of thermodynamics (5), the Cai-Kim approach, the Clausius relation and the Bekenstein entropy relation (as a candidate for the total entropy of system) to get an equation (8) which may govern the Friedmann equation (10) [9 11] .

Recently, Tsallis and et al. used the generalized nonadditive entropy to get a new relation for the Schwarzschild black hole entropy [54]. Based on their proposal, the entropy of a $3+1$ dimensional black hole $\left(S_{A}\right)$ is

$$
S_{A}=\gamma A^{\frac{3}{2}}
$$

where $\gamma$ is the proportionality constant, and should be evaluated from the dimensional analysis and other parts of physics [54]. It is worth mentioning that because entropy is a non-zero positive quantity [25], $\gamma$ should be a non-zero positive quantity. Since the apparent horizon is a holographic surface and it may play the role of boundary for cosmological setup [6 8], we generalize this entropy relation to the apparent horizon of FLRW metric and study its results. Indeed, since de-Sitter spacetime includes a static black hole, it is a static spacetime, such generalization for the de-Sitter spacetime is not far from reality. Moreover, 
it was shown that the apparent horizon entropy in radiation and dust dominated quantum universes also meets Eq. (111) [55]. Therefore, our generalization is not far-fetched. Now, by inserting this relation into Eq. (8), and using Eq. (3), we reach at

$$
\rho=\frac{9 \gamma}{\sqrt{\pi}} \sqrt{H^{2}+\frac{\kappa}{a^{2}}} .
$$

Because this equation differs from the Friedmann equation (10), we conclude that if we accept the Friedmann equation and relation (11) for the apparent horizon entropy, then relation (11) does not lead to satisfaction of the unified first law of thermodynamics. Therefore, one can blame all of the assumptions and recipe leading to Eq. (8), and also similar attempts such as those introduced in Refs. [9, 10, 16, 24]. Briefly, it seems that there is an inconsistency between the generalized entropy relation, Friedmann equation and the unified first law of thermodynamics.

It was also argued that a dark energy candidate, due to its unknown nature, may modify the apparent horizon entropy in various theories of gravity [19 23]. In fact, by decomposing $\rho$ to $\rho_{D}+\rho_{o}$, in which $\rho_{D}$ denotes the energy density of dark energy candidate and $\rho_{o}$ includes the energy density of other parts of cosmos, using the Friedman equation (10), continuity equation (4) together with the unified first law of thermodynamics and the Clausius relation, one can obtain a new relation for the horizon entropy as

$$
S_{A}=S_{B}+\frac{1}{6} \int A^{2} d \rho_{D},
$$

while dark energy does not interact with other parts of cosmos [21, 22]. Now, by combining this equation with Eq. (11), and after some calculation, we get

$$
\rho_{D}=\frac{3 H^{2}}{8 \pi}-\frac{9 \gamma}{\sqrt{\pi}} H
$$

for the energy density of dark energy candidate in a flat FLRW universe $(\kappa=0)$. It is useful to note here that there are various models for dark energy in which the density of dark energy candidate is similar to this result [65-78]. Moreover, for a FLRW universe with arbitrary curvature parameter $\kappa$, we reach

$$
\rho_{D}=\frac{3\left(H^{2}+\frac{\kappa}{a^{2}}\right)}{8 \pi}-\frac{9 \gamma}{\sqrt{\pi}} \sqrt{H^{2}+\frac{\kappa}{a^{2}}},
$$

which is also similar to some previous works [19, 79 82], and converges to Eq. (14) in the appropriate limit $(\kappa=0)$. Therefore, a dark energy candidate with profile density, satisfying Eq. (15), may be considered as an origin for the generalized entropy relation. 


\section{Some properties of the obtained dark energy candidate}

Considering $V=\frac{4 \pi}{3} \tilde{r}_{A}^{3}$, by combining Eqs. (2) and (15) with each other, we get

$$
\rho_{D}=\alpha V^{-\frac{2}{3}}+\beta V^{-\frac{1}{3}}
$$

in which $\alpha=\left(\frac{4 \pi}{3}\right)^{\frac{2}{3}} \frac{3}{8 \pi}$ and $\beta=-\left(\frac{4 \pi}{3}\right)^{\frac{2}{3}} \frac{9 \gamma}{\sqrt{\pi}}$. Bearing the $p_{D}=-\frac{\partial E_{D}}{\partial V}$ relation in mind, where $p_{D}$ and $E_{D}=\rho_{D} V$ denote the pressure and energy of dark energy candidate, respectively, simple calculation leads to

$$
p_{D}=-\frac{1}{3}\left(\rho_{D}+\beta V^{-\frac{1}{3}}\right)
$$

for the dark energy candidate pressure. For the state parameter $w_{D}=\frac{p_{D}}{\rho_{D}}$, one also reaches

$$
w_{D}=-\frac{1}{3}\left(1+\frac{\beta V^{\frac{1}{3}}}{\alpha+\beta V^{\frac{1}{3}}}\right) .
$$

It was shown that in order to describe the current acceleration phase of universe expansion $(\ddot{a} \geq 0)$, the dark energy state parameter should meet the $w_{D} \leq-\frac{1}{3}$ condition [18]. Applying this condition to Eq. (18), we get the $\gamma \geq \gamma_{0}=\frac{1}{24 \tilde{r}_{A} \sqrt{\pi}}\left(\frac{3}{4 \pi}\right)^{\frac{1}{3}}$ condition for the gamma parameter. Therefore, the minimum value of $\tilde{r}_{A}$ may be used to choose a suitable value for $\gamma$. As an example, for a flat universe of $w_{D}=-1$, it is easy to show that $\gamma_{\left[w_{D}=-1\right]}=\frac{H}{12 \sqrt{\pi}}\left(\frac{3}{4 \pi}\right)^{\frac{1}{3}}$. Since for a flat universe $\tilde{r}_{A}=\frac{1}{H}$, we can see that, as a desired result, $\gamma_{\left[w_{D}=-1\right]}>\gamma_{0}$.

\section{EMERGENCE OF SPACETIME AND THE UNIVERSE EXPANSION}

According to Verlinde's hypothesis, the tendency of systems to increase their entropy (the second law of thermodynamics) leads to the emergence of spacetime and gravity [26]. In this approach, the Bekenstein entropy and the energy definition play the key role in getting the gravitational field equations [27-48]. In addition, Padmanabhan argues that the positive difference between the surface and bulk degrees freedom may lead to emergence of spacetime and thus the universe expansion [49 53]. Although the surface degrees of freedom are proportional with the Bekenstein entropy, the role of the second law of thermodynamics is not clear in Padmanabhan's approach. In fact, based on Padmanabhan's argument $\frac{d V}{d t} \propto$ $\left(N_{s}-N_{b}\right)$ in which $N_{s}=A=4 S_{B}$ and $N_{b}=\frac{2|E|}{T}$ denote the surface and bulk degrees of freedom, respectively [49]. Moreover, $E=(\rho+3 p) V$ is the Komar mass, while $T$ is the CaiKim temperature [9, 15]. $\rho$ and $p$ are also the energy density and pressure of a source filling 
the background, respectively $\lfloor 49$ 53]. Finally, by equating the proportionality constant to one, Padmanabhan proposes [49]

$$
\frac{d V}{d t}=N_{s}-N_{b}
$$

Bearing the continuity equation (4) in mind, it is a matter of calculation to show that this hypothesis leads to the Friedman equation in various theories of gravity [49 53]. Now, focus on the generalized entropy $\left(S_{A}=3 \gamma \sqrt{4 \pi} V\right)$, we may interpret that the tendency of systems to increase their entropy (the second law of thermodynamics) implies $\dot{V} \geq 0$. Therefore, if we accept Padmanabhan's proposal $\left(\frac{d V}{d t} \propto\left(N_{s}-N_{b}\right)\right)$, we conclude that the second law of thermodynamics implies $N_{s} \geq N_{b}$. Moreover, if we take the proportionality constant equal to $3 \gamma \sqrt{4 \pi}$, we get

$$
\dot{S}_{A}=3 \gamma \sqrt{4 \pi} \frac{d V}{d t}=3 \gamma \sqrt{4 \pi}\left(N_{s}-N_{b}\right)
$$

leading to $\frac{d V}{d t}=N_{s}-N_{b}$. Based on Eq. (20), the entropy changes of system is proportional with the degrees of freedom which fall from the system surface into its volume. The number of these falling degrees of freedom is equal to the difference between the surface and bulk degrees of freedom. Finally, we see that the second law of thermodynamics implies a positive difference between the surface and bulk degrees of freedom, which leads to the emergence of spacetime and the Friedmann equations in various theories of gravity [49 53]. Briefly, we saw that the generalized entropy is in agreement with the second law of thermodynamics (Verlinde's approach) and Padmanabhan's hypothesis. Indeed, the generalized entropy may help us in making a bridge between the systems tendency to increase their entropy (the second law of thermodynamics) as the origin of the emergence of spacetime in Verlinde's hypothesis and Padmanabhan's approach.

\section{SUMMARY AND CONCLUDING REMARKS}

After referring to the Tsallis et al. proposal for the horizon entropy of the Schwarzschild black hole, we have generalized it to the apparent horizon of the FLRW universe and pointed out the inconsistency between the generalized entropy relation, the Friedmann equations and the unified first law of thermodynamics. As we have addressed, our generalization is supported by the quantum cosmology considerations [55]. Additionally, in order to eliminate 
this inconsistency, we took into account the works in which authors claim that a dark energy candidate may modify the horizon entropy and thus the Bekenstein limit in the Einstein framework. In continue, we could get an expression for the energy density of dark energy candidate. Moreover, by considering the pressure definition in thermodynamics, we got a relation for the pressure and state parameter of the obtained dark energy candidate. Our study shows that this dark energy candidate may be used to describe the current phase of universe expansion. Finally, we showed that the new proposal for the horizon entropy may be used to make a bridge between the tendency of systems to increase their entropy (the second law of thermodynamics) as the backbone of Verlinde's proposal, and the positive difference between the surface and bulk degrees of freedom as the origin of the spacetime emergence and its expansion in Padmanabhan's approach. The latter helps us in getting more close to the thermodynamic roots of Padmanabhan's approach. Briefly, our investigation shows that the generalized entropy may help us in taking into account the second law of thermodynamics as the cause of positive difference between the surface and bulk degrees of freedom. In fact, by combining the generalized entropy relation, the second law of thermodynamics and Padmanabhan's proposal with each other, we saw that the volume changes of system is equal to the difference between the surface and bulk degrees of freedom. The spacetime emerges with the fall of these degrees of freedom from the system surface into its volume, a mechanism which may guarantee the universe expansion.

\section{Acknowledgments}

This work has been supported financially by Research Institute for Astronomy \& Astrophysics of Maragha (RIAAM).

[1] E. Poisson, A Relativist's Toolkit (Cambridge University Press, UK, 2004)

[2] T. Padmanabhan, Phys. Rept. 406, 49 (2005)

[3] T. Jacobson, Phys. Rev. Lett. 75, 1260 (1995)

[4] C. Eling, R. Guedens, T. Jacobson, Phys. Rev. Lett. 96, 121301 (2006)

[5] M. Roos, Introduction to Cosmology (John Wiley and Sons, UK, 2003)

[6] S. A. Hayward, S. Mukohyana, M.C. Ashworth, Phys. Lett. A 256, 347 (1999) 
[7] S. A. Hayward, Class. Quantum Grav. 15, 3147 (1998)

[8] D. Bak, S. J. Rey, Class. Quantum Grav. 17, 83 (2000)

[9] R. G. Cai, S. P. Kim, JHEP 0502, 050 (2005)

[10] M. Akbar, R. G. Cai, Phys. Rev. D 75, 084003 (2007)

[11] M. Akbar, R. G. Cai, Phys. Lett. B 635, 7 (2006)

[12] A. Sheykhi, B. Wang, R. G. Cai, Nucl. Phys. B 779, 1 (2007)

[13] A. Sheykhi, B. Wang, R. G. Cai, Phys. Rev. D 76, 023515 (2007)

[14] H. Moradpour, Phys. Lett. B 757, 187 (2016)

[15] R. G. Cai, L.M. Cao, Y. P. Hu, Class. Quantum. Grav. 26, 155018 (2009)

[16] D. W. Tian, I. Booth, Phys. Rev. D 92, 024001 (2015)

[17] H. Moradpour, A. Sheykhi, N. Riazi, B. Wang, AHEP. 2014, 718583 (2014)

[18] H. Moradpour and N. Riazi, Int. J. Theor. Phys. 55, 268 (2016)

[19] C. J. Feng, X.Z. Li, X.Y. Shen, Mod. Phys. Lett. A 27, 1250182 (2012)

[20] A. Sheykhi, Can. J. Phys 92, 529 (2014)

[21] H. Ebadi, H. Moradpour, Int. J. Mod. Phys. D 24, 1550098 (2015)

[22] H. Moradpour, R. Dehghani, arXiv:1512.00366 (Accepted by AHEP)

[23] H. Moradpour, M. T. Mohammadi Sabet, Can. J. Phys. 94, 1 (2016)

[24] A. Awad, A. F. Ali, JHEP 06, 093 (2014)

[25] H.B. Callen, Thermodynamics and Introduction to Thermostatics (New York: John Wiley and Sons, 1985)

[26] E. Verlinde, JHEP 1104, 029 (2011).

[27] R. G. Cai, L. M. Cao, N. Ohta, Phys. Rev. D 81, 061501(R) (2010).

[28] R. G. Cai, L. M. Cao, N. Ohta, Phys. Rev. D 81, 084012 (2010).

[29] L. Smolin, arXiv:1001.3668.

[30] M. Li, Y. Wang, Phys. Lett. B 687, 243 (2010).

[31] Y. Tian, X. Wu, Phys. Rev. D 81, 104013 (2010).

[32] Y. S. Myung, arXiv:1002.0871.

[33] I. V. Vancea, M. A. Santos, arXiv:1002.2454.

[34] L. Modesto, A. Randono, arXiv:1003.1998.

[35] A. Sheykhi, Phys. Rev. D 81, 104011 (2010).

[36] B. Liu, Y. C. Dai, X. R. Hu, J. B. Deng, Mod. Phys. Lett. A 26, 489 (2011). 
[37] S. H. Hendi, A. Sheykhi, Phys. Rev. D 83, 084012 (2011).

[38] A. Sheykhi, S. H. Hendi, Phys. Rev. D 84, 044023 (2011).

[39] S. H. Hendi, A. Sheykhi, Int. J Theor. Phys. 51, 1125 (2012).

[40] A. Sheykhi, Z. Teimoori, Gen. Relativ. Gravit. 44, 1129 (2012).

[41] Y. Ling, J. P. Wu, J. Cosmol. Astropart. Phys. 1008, 017 (2010).

[42] A. Sheykhi, Int. J Theor. Phys. 51, 185 (2012).

[43] W. Gu, M. Li, R. X. Miao, arXiv:1011.3419.

[44] R. X. Miao, J. Meng, M. Li, arXiv:1102.1166.

[45] Y. X. Liu, Y. Q. Wang, S. W. Wei, Class. Quantum Grav. 27, 185002 (2010).

[46] R. B. Mann, J. R. Mureika, Phys. Lett. B 703, 167 (2011).

[47] A. Sheykhi, H. Moradpour, N. Riazi, Gen. Rel. Grav. 45, 1033 (2013).

[48] H. Moradpour, A. Sheykhi, Int. J. Theo. Phys. DOI: 10.1007/s10773-015-2756-2.

[49] T. Padmanabhan. arXiv:1206.4916 (2012)

[50] A. Sheykhi, Phys. Rev. D 87, 061501(R) (2013)

[51] A. Sheykhi, M. H. Dehghani, S. E. Hosseini, Phys. Lett. B 726, 23 (2013)

[52] Y. Ling, W. J Pan. Phys. Rev. D 88, 043518 (2013)

[53] M. Hashemi, S. Jalalzadeh, S. Vasheghani Farahani, Gen. Relativ. Gravit. 47, 139 (2015)

[54] C. Tsallis, L. J. L. Cirto, Eur. Phys. J. C 73, 2487 (2013)

[55] M. Rashki, S. Jalalzadeh, Phys. Rev. D 91, 023501 (2015)

[56] Komatsu, Nobuyoshi, Shigeo Kimura. Phys. Rev. D 88, 083534 (2013)

[57] Komatsu, Nobuyoshi, Shigeo Kimura. Phys. Rev. D 89, 123501 (2014)

[58] Komatsu, Nobuyoshi, Shigeo Kimura. Phys. Rev. D 90, 123516 (2014)

[59] Abreu, Everton MC, Jorge Ananias Neto. Phys. Lett. B 727, 524 (2013)

[60] E. M. C. Abreu, J. Ananias Neto, A. C. R. Mendes, W. Oliveira, Physica. A 392, 5154 (2013)

[61] Barboza, Edsio M., et al., Physica. A 436, 301 (2015)

[62] Nunes, Rafael C., et al., arXiv:1509.05059 (2015)

[63] K. Nobuyoshi and S. Kimura, arXiv:1511.04364 (2015)

[64] O. Kamel and M. Tribeche, Ann. Phys. 342, 78 (2014)

[65] G. Veneziano, Nucl. Phys. B 159, 213 (1979)

[66] E. Witten, Nucl. Phys. B 156, 269 (1979)

[67] C. Rosenzweig, J. Schechter and C. G. Trahern, Phys. Rev. D 21, 3388 (1980) 
[68] V. Sahni and A. Starobinsky, Int. J. Mod. Phys. D9, 373 (2000)

[69] I. L. Shapiro and J. Solà, JHEP. 0202, 006 (2002)

[70] J. Grandea, J. Solà and H. Stefancic, Phys. Let. B 12, 040 (2006)

[71] J. Grandea, J. Solà and H. Stefancic, JCAP 0608, 011 (2006)

[72] F. R. Urban and A. R. Zhitnitsky, Phys. Lett. B 688, 9 (2010)

[73] J. Solà, Jour. Phys. Conf. Seri. 283, 012033 (2011)

[74] M. Maggiore, L. Hollenstein, M. Jaccard and E. Mitsou, Phys. Lett. B 704, 102 (2011)

[75] M. Maggiore, Phys. Rev. D 83, 063514 (2011)

[76] N. Ohta, Phys. Lett. B 695, 41 (2011)

[77] A. R. Zhitnitsky, Phys. Rev. D 86, 045026 (2012)

[78] J. A. S. Lima, S. Basilakos and J. Solà, Mont. Not. R. Astron. Soc. 431, 923 (2013)

[79] I. L. Shapiro, J. Solà and H. Stefancic, JCAP 0501, 012 (2005)

[80] J. Solà, J. Phys. A 41, 164066 (2008)

[81] J. Grandea and A. Pelinson, J. Solà, Phys. Rev. D 79, 043006 (2009)

[82] A. Sheykhi and B. Wang. Mod. Phys. Lett. A25, 1199 (2010) 